\title{
EDITORIAL \\ Can big data bridge the chasm? Issues, opportunities, and strategies for the evolving value-based health care environment
}

\author{
Christopher M. Holland, MD, PhD, ${ }^{1}$ Kevin T. Foley, MD, ${ }^{2}$ and Anthony L. Asher, MD ${ }^{3}$ \\ ${ }^{1}$ Department of Neurosurgery, Emory University, Atlanta, Georgia; '2Department of Neurosurgery, Semmes Murphey Clinic, \\ Memphis, Tennessee; and ${ }^{3}$ Department of Neurosurgery, Carolina Neurosurgery \& Spine Associates, and Neuroscience \\ Institute, Carolinas Healthcare System, Charlotte, North Carolina
}

$\mathrm{T}$ He practice of modern medicine necessitated the collection and interpretation of individualized data in the context of normative values and ranges derived from populations. As the methods for recording and storing these data points changed from analog paper charts to digital archives, the volume of available information has grown exponentially. These extremely large data sets are referred to colloquially as "big data" and are not unique to health care, but rather have become ubiquitous in all fields and industries. In many domains, but in health care in particular, these large data repositories hold enormous opportunity for improvement.

Many devices facilitate the automatic measurement and storage of data points at high frequencies. For example, whereas a patient may have had their heart rate recorded only a couple of times per year during office visits with their physician, it is now possible to capture and record this information continuously, resulting in thousands of data points in a single day. The ability to collect these incredible amounts of data has far outpaced our ability to analyze it. It is in the meaningful utilization, analysis, and interpretation of these vast quantities of data that true value will be found and the digital transformation of medicine will occur.

Robust tools for the analysis of big data have already been developed for and implemented in several areas of medicine, including genomics, using next-generation sequencing (NGS) technologies and high-throughput screening (HTS) of novel pharmaceuticals. The meaningful utilization of big data is driving the personalization of medicine, wherein diagnoses are made, treatment regimens designed, and prognoses forecast based on comprehensive analyses of individuals, not on population-based research.

In addition to genomic analysis and pharmaceutical development, big data is manifest in many other areas of medicine including the widespread implementation of electronic health records (EHRs); smart mobile technologies used for informational, diagnostic, or health maintenance applications; administrative and financial databases repurposed for resource utilization assessment; clinical trial databases; and the many evolving prospective clinical data registries. These diverse sources are increasingly being leveraged to drive personalized and precision medicine. In addition, many of these data sources are being exploited as baselines for the assessment of quality for clinical and financial purposes.

As the health care landscape shifts from fee-for-service (FFS) to value-based care, subspecialty medicine, and especially the surgical specialties, will be required to report and be held accountable to generic and nonspecific quality measures in the absence of specialty-specific metrics. Existing generic measures not only have little relevance to specialty clinical practice, but also fail to meaningfully correlate with patient outcomes. Private payers, following the lead of the Centers for Medicare and Medicaid Services (CMS), have been and will continue to use available measures to impose financial repercussions on providers and provider groups.

This is the changing milieu in which neurological sur- 
gery can and must take an active role in the development of meaningful quality measures and instruments that reflect all components of the neurosurgical care continuum through the effective utilization of big data. A multitude of regulatory and legislative requirements have created a complicated system of quality programs. The authorization of alternative mechanisms for quality reporting, including the Physician Quality Reporting System (PQRS) Qualified Clinical Data Registry (QCDR), now empowers surgical specialties to create and use highly granular registries. Along these lines, neurosurgery has led the development of the first specialty-specific QCDR by leveraging our experience with the National Neurosurgery Quality and Outcomes Database ( $\left.{ }^{2} \mathrm{QOD}\right){ }^{1}$

Imperfect quality data are not only being used to increase or reduce payments and fees. The recent ProPublica data release highlights another critical issue in the quantification of medical performance with imperfect measures (https://projects.propublica.org/surgeons/). The reliance on indices such as complication rates in the absence of risk adjustment has the potential to unfairly penalize surgeons who assume risk in their practices through the care of the sickest patients with significant comorbidities and the most complicated surgical problems. We must all work to ensure a health care system that rewards rather than penalizes those who care for our most vulnerable patients.

When used in an appropriate manner, the direct measurement and quantification of quality in a clinically relevant way can better inform our patients about potential interventions for shared decision making in accordance with the directives of patient-centered care. In addition, quality improvement initiatives facilitate practice-based learning and effective resource utilization. Understanding the true value of neurosurgical interventions is predicated on the use of real-world data from clinical practice as opposed to data collected through clinical trials, which is rarely representative of "real-world" patient care. Clinical registries are an important big data instrument and are ideal for the aggregation of meaningful information, despite the present burdens of data collection. They have multiple advantages for advancing medical science including scalability, cost-effectiveness, and strong external validity (the latter characteristic correlating with data "generalizability"). Increasingly, clinical registries are being applied for patient profiling, care redirection, reimbursement adjustment, and comparative effectiveness research.

The development of tools and statistical methods with the capacity for parsing and analyzing the vast amounts of available medical data has significantly lagged behind the mechanisms for collecting and aggregating these data. However, one such method, propensity scoring, holds the promise of increasing the generalizability and value of observational data by reducing treatment bias. It is highly likely that advanced statistical techniques will be increasingly used to augment the value of observational data. In fact, well-designed registry trials may approximate the results of randomized controlled trials on the same topic. ${ }^{2}$ Some have gone so far as to characterize registry-based trials as the next "disruptive technology" in clinical research. ${ }^{3,4}$

We as neurosurgeons now stand at the precipice of the chasm dividing the familiar FFS environment and the uncertain world of value-based care. Although the development of the first QCDR for neurosurgery is a major achievement and paves the way for the creation of additional subspecialty-specific registries, neurosurgeons must acquire the knowledge and skill sets to thrive in the era of big data. These skills include an understanding of and familiarity with data architecture and analytical platforms and tools; a knowledge of patient-centered, value-based care; and also basic and advanced information technology skills.

The future for neurosurgery is bright, particularly for those who accept the challenge of using data, both big and small. Importantly, we must invest ourselves in efforts to accurately assess quality in order to demonstrate the value of neurosurgical interventions, and we must conduct quality improvement programs to significantly impact our patient outcomes and help create a meaningful and sustainable health care system.

http://thejns.org/doi/abs/10.3171/2015.9.FOCUS15497

\section{References}

1. Asher AL, McCormick PC, Selden NR, Ghogawala Z, McGirt MJ: The National Neurosurgery Quality and Outcomes Database and NeuroPoint Alliance: rationale, development, and implementation. Neurosurg Focus 34(1):E2, 2013

2. Benson K, Hartz AJ: A comparison of observational studies and randomized, controlled trials. N Engl J Med 342:18781886,2000

3. Dehabreh IJ, Kent DM: Can the learning healthcare system be educated with observational data? JAMA 312:129-130, 2014

4. Lauer MS, D'Agostino RB: The randomized registry trialthe next disruptive technology in clinical research? N Engl J Med 369:1579-1581, 2013 